\title{
Evaluación de las Propiedades Mecánicas de una Mezcla Densa en Caliente Modificada con un Desecho de PVC
}

\author{
Hugo A. Rondón-Quintana ${ }^{1}$ \\ Fredy A. Reyes-Lizcano ${ }^{2}$
}

\section{Resumen}

El presente trabajo evaluó en laboratorio, la resistencia mecánica bajo carga monotónica, el módulo resiliente y la resistencia a la deformación permanente que experimenta una mezcla asfáltica cuando se modifica con un desecho de policloruro de vinilo (PVC) por vía húmeda. Adicionalmente, fue evaluada durante 21 meses, la influencia del medio ambiente de la ciudad de Bogotá D.C. sobre las propiedades mecánicas de la mezcla modificada. De los resultados obtenidos se concluye que la resistencia mecánica de la mezcla asfáltica modificada es superior en comparación con la convencional. La tendencia general de las mezclas con el tiempo de exposición al medio ambiente de Bogotá D.C. es experimentar un aumento en los valores de rigidez debido principalmente a procesos de endurecimiento por envejecimiento del ligante asfáltico. Sin embargo, para el caso de las mezclas fabricadas con CA 60-70 y modificadas con PVC en los primeros 15 meses de exposición, la rigidez disminuye por efecto de micro-fisuración térmica.

\section{Palabras clave}

Asfalto modificado, mezcla densa en caliente, desecho de PVC, propiedades mecánicas.

1 Facultad del Medio Ambiente y Recursos Naturales, Universidad Distrital Francisco José de Caldas, Bogotá-Colombia, harondonq@udistrital.edu.co

2 Facultad de Ingeniería, Departamento de Ingeniería Civil, Pontificia Universidad Javeriana, Bogotá-Colombia, fredy.reyes@javeriana.edu.co

Fecha de recepción: 07 de septiembre de 2010

Fecha de aceptación: 20 de septiembre de 2011

Rev. Tecno Lógicas No. 27, ISSN 0123-7799, Diciembre de 2011, pp. 11-31 


\section{Abstract}

The strength under monotonic load, resilient modulus and rutting were evaluated on a hot asphalt mixture modified with a residue of polyvinyl chloride (PVC) by wet way. Additionally, the influence of environmental conditions of Bogotá D.C. was evaluated during 21 months on mechanical properties of modified asphalt mixture. The results show that the mechanical properties evaluated were higher for the MDC-2 mixes modified with PVC compared with mixtures with asphalts without additives. The PVC produces higher penetration resistance and lower thermal flow susceptibility. The general tendency of the mixtures is increase the modulus with time due to aging of the asphalt cement. However, mixtures with AC 60-70 and modified with PVC decrease stiffness in the first months due to thermal micro-cracks phenomena.

\section{Keywords}

Modified asphalt, hot dense mix, polyvinyl chloride, mechanical properties. 


\section{INTRODUCCIÓN}

En Colombia, la tendencia del parque automotor en los últimos 30 años ha sido incrementar en número y magnitud de cargas (Ministerio de Transporte, 2004, 2006). Lo anterior genera en las capas asfálticas mayores magnitudes de esfuerzo y deformación. Estos mayores niveles de carga deben ser contrarrestados con materiales asfálticos que presenten mejores comportamientos que los tradicionales. Con el fin de mejorar las propiedades de los materiales asfálticos y las mezclas asfálticas, se han adelantado diversas investigaciones en Colombia y el mundo (Papagiannakis\&Lougheed, 1995; Copeland et al., 2007; Rondón et al., 2008). El objetivo principal de estas investigaciones ha sido mejorar las propiedades mecánicas, químicas y reológicas de los asfaltos y las mezclas asfálticas convencionales (mezcla que emplea asfaltos sin ningún aditivo) con el fin de modificar la rigidez, la resistencia bajo carga monotónica, al ahuellamiento, a la fatiga, al envejecimiento, y disminuir la susceptibilidad térmica.

La mayor parte de las investigaciones realizadas en el área de los asfaltos modificados utilizan como agentes modificadores polímeros del tipo elastómero (McQuillen et al., 1998; Stastna et al., 2000; 2003; Chen et al., 2002; Lee et al., 2008; Instituto de Desarrollo Urbano, 2009; Olivares et al., 2009). Este tipo de aditivos al ser agregados al asfalto mejoran principalmente el comportamiento resiliente (recuperación elástica) de las mezclas cuando son solicitadas a ciclos de carga y descarga especialmente a altas temperaturas de servicio. Como ya se mencionó, en este estudio se propone modificar el cemento asfáltico con un desecho polimérico del tipo plastomérico como es el PVC. Este aditivo se escogió debido principalmente a que este tipo de polímero (plastómero) generalmente produce un incremento en la resistencia mecánica de las mezclas a altas temperaturas ya que el asfalto se rigidiza, es un material de alta resistencia térmica y química cuya degradación natural dura más de 100 años (CAG, 2009), y de acuerdo con Reyes \& Figueroa (2008), en Bogotá D.C. diariamente se producen alrededor de 600 toneladas de basuras de las cuales el 10\% aproximadamente son plásticos y el consumo per cápita de estos materiales en Colombia es de $11.3 \mathrm{~kg}$ anuales. De este $10 \%$ gran parte 
proviene de desechos de PVC los cuales pueden ser utilizados para modificar las propiedades de mezclas asfálticas y así disminuir el impacto ambiental negativo que generan.

Por otro lado, los principales factores que afectan la durabilidad de mezclas asfálticas, asumiendo que se encuentran bien construidas son la edad de envejecimiento y el daño por humedad (Airey, 2003). Es decir, una mezcla asfáltica debe ser diseñada y construida no solo para que resista las cargas impuestas por el tránsito sino también la acción del medio ambiente. Cuantificar la influencia que tiene el ambiente sobre el comportamiento de este tipo de material no es una tarea fácil. La forma como se realiza actualmente es separando cada uno de los componentes que lo conforman (agua, temperatura y rayos ultra-violeta entre otros) y evaluar la influencia de cada uno de ellos (desde el punto de vista mecánico y químico) de manera separada sobre el ligante y las mezclas (Kemp\&Predoehl, 1981; Welborn, 1984; Kim et al., 1987; Airey, 2003; Said, 2005; Shen et al., 2006).

Adicionalmente, por lo general estos estudios utilizan ensayos y equipos (hornos para ensayo de película delgada del tipo TFOT o rotatorios RTFOT, microondas, vasijas de envejecimiento de ligantes a presión PAV) que no pueden reproducir totalmente la influencia que tiene cada uno de los componentes del ambiente sobre las propiedades mecánicas y reológicas de las mezclas y los ligantes asfálticos (Jemison et al., 1991; Verhasselt, 1997; Migliori\& Corté, 1998). Incluso la mayor parte de las investigaciones combinan estos ensayos y equipos para intentar evaluar la forma como cambian las propiedades de estos materiales cuando experimentan condiciones reales del ambiente (Jemison et al., 1991; Migliori\& Corté, 1998; Airey, 2003). En conclusión general se reporta que estas metodologías de ensayo tienen como limitación principal que son incapaces aún de reproducir las condiciones reales a las cuales están expuestas las mezclas asfálticas in situ. Una forma de medir in situ la influencia que tiene el ambiente en el comportamiento de mezclas asfálticas es realizando tramos de prueba o pistas de prueba a escala. La limitación de este tipo de pruebas radica en que son costosas y en ellas no se puede medir de manera directa y por separado, la influencia que tienen las cargas vehiculares y el ambiente. 
El artículo presenta los resultados experimentales de ensayar una mezcla asfáltica densa en caliente tipo MDC-2 (INVIAS, 2007a) modificada con un desecho de PVC por vía húmeda. Por vía húmeda el PVC es adicionado a alta temperatura con el cemento asfáltico y luego este ligante modificado se agrega a los pétreos para conformar la mezcla asfáltica tipo MDC-2. Para la elaboración de las mezclas y la evaluación de las mismas, fueron modificados los dos tipos de cementos asfálticos (CA) fabricados en Colombia: CA 80-100 y CA 60-70 (se refieren a CA cuya medida en el ensayo de penetración ASTM D-5 está en un rango entre 80 a 100 y 60 a 70 décimas de $\mathrm{mm}$ respectivamente). Estos ligantes son los utilizados en Colombia cuando la temperatura media anual promedio de la zona donde se construirá la capa asfáltica es inferior (CA 80-100) y superior (CA 60-70) a $24^{\circ} \mathrm{C}$ (INVIAS, 2007a). El $\mathrm{PVC}$ se adicionó al CA por vía húmeda.

Para la evaluación de la resistencia mecánica bajo carga monotónica de las mezclas asfálticas convencionales y modificadas se empleó el ensayo Marshall y para el CA con y sin aditivo se realizaron ensayos de penetración a diferentes temperaturas y punto de ablandamiento. Con el fin de evaluar el comportamiento de las mezclas bajo carga cíclica se realizaron ensayos de módulo resiliente y deformación permanente medida en la dirección vertical. Adicionalmente el artículo presenta la influencia que tienen las condiciones ambientales de la ciudad de Bogotá D.C. (Colombia) sobre las propiedades mencionadas. Se optó por analizar mezclas tipo MDC-2 debido a que son las más utilizadas en Colombia para conformar capas de rodadura las cuales son las que se encuentran sometidas de manera directa a las condiciones del ambiente.

De la misma forma, las condiciones climáticas de Bogotá D.C. fueron escogidas debido principalmente a que la ciudad se encuentra en una zona donde se presentan las siguientes condiciones ambientales en un día determinado: clima predominantemente frío con temperaturas mínimas y máximas promedio de $6^{\circ} \mathrm{C}$ y $20^{\circ} \mathrm{C}$ respectivamente, humedad entre el 60 al 100\%, presencia de lluvias periódicas en cualquier momento del día, precipitación diaria promedio entre 2,06 y $9,03 \mathrm{~cm}$. y altura sobre el nivel del mar de $2640 \mathrm{~m}$. Con el fin de entender con mayor precisión la influencia del ambiente sobre el comportamiento de las mezclas asfálticas 
analizadas en el presente estudio, en futuras investigaciones se analizarán otros climas diferentes. En este artículo se presentarán los resultados de los primeros 21 meses del proyecto. En la fabricación de las piezas tipo marco de motocicleta, la verificación metrológica es realizada en mesas de medición con calibres diseñados específicamente para los diferentes subtipos de las piezas tipo marco. A pesar de ser un procedimiento eficiente, requiere de un permanente control sobre los calibres, los cuales deben ser verificados (dimensionalmente) y ajustados regularmente.

\section{METODOLOGíA}

\subsection{Caracterización de Materiales}

En la Tabla 1 se presentan los valores obtenidos de los ensayos de caracterización al agregado pétreo. Se observa en esta tabla que los valores de cada uno de los ensayos cumplen con el requisito mínimo de calidad exigido por las especificaciones INVIAS (2007a) para fabricar mezclas tipo MDC-2. Para cumplir con las especificaciones del INVIAS (2007a), se modificó la granulometría original de los agregados, tomando como referencia los valores promedios en porcentajes de la franja granulométrica que exige la especificación para la elaboración de las mezclas tipo MDC-2. A los cementos asfálticos se les realizaron los ensayos típicos que exige la especificación INVIAS (2007a) para caracterizarlos y los resultados se presentan en las Tablas 2 y 3 . El policloruro de vinilo (PVC) utilizado es un desecho obtenido de la producción de resinas prime de PVC, presenta una densidad de $0,9 \mathrm{~g} / \mathrm{cm}^{3}$ y partículas de coloración blanca que pasan el tamiz No. 200 en un ensayo de granulometría cuando el material está en estado seco.

\subsection{Diseño de Mezclas Asfálticas}

Luego de realizar los ensayos al agregado pétreo y a los ligantes asfálticos se fabricaron cinco briquetas (compactadas a 75 golpes por cara) para cada porcentaje de asfalto entre 4,5 y $6,5 \%$, con el fin de realizar el diseño Marshall para determinar el conte- 
nido óptimo de asfalto de las mezclas convencionales. Los porcentaje óptimos de cemento asfáltico son de 5,3\% y 5,6\% para el caso de mezclas fabricadas con CA 80-100 y CA 60-70 respectivamente. En estos porcentajes se están cumpliendo los requisitos mínimos exigidos por la especificación INVIAS (2007a) para MDC-2 y tránsitos tipo NT1 y/o NT2 (bajos y medios volúmenes de tránsito).

Tabla 1. Caracterización del agregado pétreo. Fuente: Autores

\begin{tabular}{lc}
\multicolumn{1}{c}{ Ensayo } & Resultado \\
\hline Peso específico & 2,56 \\
Equivalente de arena & $86 \%$ \\
Caras fracturadas & $95 \%$ \\
Índice de alargamiento & $9,2 \%$ \\
Índice de aplanamiento & $9,5 \%$ \\
Ataque en Sulfato de sodio & $12,4 \%$ \\
Microdeval & $20,3 \%$ \\
Resistencia al desgaste & $23,5 \%$ \\
máquina de los Ángeles & \\
\hline
\end{tabular}

Tabla 2. Características generales del cemento asfáltico CA 80-100.

Fuente: Autores

\begin{tabular}{|c|c|c|c|c|}
\hline Ensayo & Método & Unidad & CA $80-100$ & Resultado \\
\hline \multicolumn{5}{|c|}{ Ensayos sobre el asfalto original } \\
\hline Penetración $\left(25^{\circ} \mathrm{C}, 100 \mathrm{~g}, 5 \mathrm{~s}\right)$ & ASTM D-5 & $0,1 \mathrm{~mm}$ & $80-100$ & 85 \\
\hline Índice de penetración & INV. E-724 & - & $-1 /+1$ & $-0,5$ \\
\hline Viscosidad absoluta $\left(60^{\circ} \mathrm{C}\right)$ & ASTM D-4402 & Poises & 1000 mín. & 1400 \\
\hline Ductilidad $\left(25^{\circ} \mathrm{C}, 5 \mathrm{~cm} / \mathrm{min}\right)$ & ASTM D-113 & $\mathrm{cm}$ & 100 mín. & $>105$ \\
\hline Solubilidad en Tricloroetileno & ASTM D-2042 & $\%$ & 99 mín. & $>99$ \\
\hline Contenido de agua & ASTM D-95 & $\%$ & 0,2 máx. & $<0,2$ \\
\hline Punto de inflamación COC & ASTM D-92 & ${ }^{\circ} \mathrm{C}$ & 232 mín. & 295 \\
\hline \multicolumn{5}{|c|}{ Ensayos sobre el residuo luego del RTFOT } \\
\hline Pérdida de masa & ASTM D-2872 & $\%$ & 1,0 máx. & 0,2 \\
\hline Penetración $\left(25^{\circ} \mathrm{C}, 100 \mathrm{~g}, 5 \mathrm{~s}\right)$ & ASTM D-5 & $\%$ & 48 mín. & 65 \\
\hline
\end{tabular}



con un Desecho de PVC

Tabla 3. Características generales del cemento asfáltico CA 60-70. Fuente: Autores

\begin{tabular}{|c|c|c|c|c|}
\hline Ensayo & Método & Unidad & CA $60-70$ & Resultado \\
\hline \multicolumn{5}{|c|}{ Ensayos sobre el asfalto original } \\
\hline Penetración $\left(25^{\circ} \mathrm{C}, 100 \mathrm{~g}, 5 \mathrm{~s}\right)$ & ASTM D-5 & $0,1 \mathrm{~mm}$ & $60-70$ & 67 \\
\hline Índice de penetración & INV. E-724 & - & $-1 /+1$ & $-0,7$ \\
\hline Viscosidad absoluta $\left(60^{\circ} \mathrm{C}\right)$ & ASTM D-4402 & Poises & 1500 mín. & 1750 \\
\hline Ductilidad $\left(25^{\circ} \mathrm{C}, 5 \mathrm{~cm} / \mathrm{min}\right)$ & ASTM D-113 & $\mathrm{cm}$ & 100 mín. & $>105$ \\
\hline Solubilidad en Tricloroetileno & ASTM D-2042 & $\%$ & 99 mín. & $>99$ \\
\hline Contenido de agua & ASTM D-95 & $\%$ & 0,2 máx. & $<0,2$ \\
\hline Punto de inflamación COC & ASTM D-92 & ${ }^{\circ} \mathrm{C}$ & 232 mín. & 275 \\
\hline \multicolumn{5}{|c|}{ Ensayos sobre el residuo luego del RTFOT } \\
\hline Pérdida de masa & ASTM D-2872 & $\%$ & 1,0 máx. & 0,4 \\
\hline Penetración $\left(25^{\circ} \mathrm{C}, 100 \mathrm{~g}, 5 \mathrm{~s}\right)$ & ASTM D-5 & $\%$ & 48 mín. & 70 \\
\hline
\end{tabular}

\subsection{Fase Experimental}

Una vez se obtuvo el porcentaje óptimo de CA, se fabricaron nuevas briquetas agregando por vía húmeda el PVC en porcentajes de 0,$5 ; 1,0$ y $1,5 \%$ (con respecto al peso total de la briqueta de $1200 \mathrm{~g}$ ), manteniendo el porcentaje de CA. Por cada porcentaje de aditivo se fabricaron cinco briquetas para ensayarlas en el aparato Marshall con el fin de evaluar la respuesta que experimentan las mezclas bajo carga monotónica en tracción indirecta. Además se realizó el mismo estudio aumentando y rebajando el porcentaje óptimo de $\mathrm{CA}$ en $0,3 \%$. La temperatura de mezclado del CA con el $\mathrm{PVC}$ fue de $155^{\circ} \mathrm{C}$. Esta temperatura fue escogida debido a que por encima de la misma el CA experimenta envejecimiento por pérdida de componentes químicos por oxidación, y por debajo, el mezclado se dificulta especialmente cuando el contenido de PVC es alto. El tiempo de mezclado del CA con el PVC fue de 45 minutos para porcentajes de adición de PVC de 0,5 y 1,0\% y de una hora para $1,5 \%$. Esta temperatura y tiempos de mezcla son similares a aquellos reportados por otros investigadores cuando han modificado CA con aditivos plastoméricos (Fang et al., 2008). Con el fin de determinar las temperaturas de mezcla y compactación para la fabrica- 
ción de las mezclas fueron ejecutados ensayos de viscosidad sobre los ligantes modificados (Tabla 4).

Tabla 4. Temperaturas de mezcla y compactación. Fuente: Autores

\begin{tabular}{ccccc}
\hline \multirow{2}{*}{$\begin{array}{c}\text { PVC } \\
{[\%]}\end{array}$} & $\begin{array}{c}\text { Temperatura de } \\
\text { mezcla }\left[{ }^{\circ} \mathrm{C}\right]\end{array}$ & $\begin{array}{c}\text { Temperatura de } \\
\text { compactación }\left[{ }^{\circ} \mathrm{C}\right]\end{array}$ & $\begin{array}{c}\text { Temperatura de } \\
\text { mezcla }\left[{ }^{\circ} \mathrm{C}\right]\end{array}$ & $\begin{array}{c}\text { Temperatura de } \\
\text { compactación }\left[{ }^{\circ} \mathrm{C}\right]\end{array}$ \\
\hline 0,0 & 135 & 130 & 142 & 134 \\
0,5 & 153 & 147 & 161 & 155 \\
1,0 & 166 & 161 & 171 & 166 \\
1,5 & 185 & 178 & 190 & 182 \\
\hline
\end{tabular}

Con los resultados obtenidos del ensayo Marshall se determinó un porcentaje de cemento asfáltico y PVC con base principalmente en el máximo valor de la relación estabilidad - flujo $E / F$ (llamada por algunos investigadores como rigidez Marshall; esta relación físicamente puede ser entendida como una resistencia mecánica evaluada en el estado de falla de las mezclas, bajo carga monotónica en un ensayo de tracción indirecta), el flujo, módulo resiliente y en los resultados de los ensayos de viscosidad. Con el valor de CA y PVC se fabricaron nuevas briquetas para realizar los ensayos de módulo resiliente (INV. E 749, INVIAS, 2007b) y resistencia a la deformación permanente de mezclas asfálticas (EN 12697-22, CEN, 2005). El módulo resiliente fue obtenido bajo tres temperaturas $\left(10,20\right.$ y $\left.30^{\circ} \mathrm{C}\right)$ y frecuencias de carga diferentes $(2,5 ; 5,0$ y $10,0 \mathrm{~Hz}$ ) utilizando un equipo Nottingham Asphalt Tester (NAT). Tres muestras y ensayos por temperatura y frecuencia de carga fueron ejecutados para medir el módulo resiliente. Las muestras para determinar el módulo fueron ensayadas bajo carga cíclica en tracción indirecta. El ensayo de resistencia a la deformación permanente bajo carga repetida fue realizado bajo un esfuerzo de 100 $\mathrm{kPa}$ y a 3600 ciclos de carga siguiendo el procedimiento normalizado por EN 12697-22 (CEN, 2005). Tres muestras fueron ensayadas por cada relación de PVC y CA para la ejecución del ensayo de deformación permanente.

Para evaluar la influencia del medio ambiente se elaboraron 180 briquetas de mezcla asfáltica MDC-2 por tipo de CA, para exponerlas al ambiente de la ciudad de Bogotá D.C., utilizando el 
contenido óptimo de CA para el caso de las mezclas convencionales y de CA y PVC para las mezclas modificadas. Este contenido fue obtenido con base en los ensayos de caracterización monotónica y dinámica. Estas muestras fueron colocadas en el techo de un edificio de la ciudad de Bogotá D.C. Durante los primeros 21 meses del proyecto, a las briquetas se les realizaron ensayos de módulo resiliente y resistencia a la deformación permanente para evaluar la evolución de estos parámetros con el tiempo de exposición al medio ambiente. Con los resultados de estos ensayos se desarrolló una ecuación empírica para predecir la evolución de la rigidez de la mezcla analizada con el tiempo de exposición al medio ambiente.

\section{RESULTADOS}

\subsection{Propiedades Bajo Carga Monotónica}

Los mayores valores de estabilidad se obtienen cuando se adiciona 1,0\% de PVC al contenido óptimo de CA (Fig. 1 y 2). De la misma forma, los mayores valores de resistencia mecánica bajo carga monotónica (evaluada a través de la relación $E / F$ ) se obtienen cuando se adiciona $0,5 \%$ de PVC al contenido óptimo CA. Los incrementos de estabilidad y $E / F$ en estos porcentajes son de $68 \%$ y $92 \%$ para CA $80-100$ y de $50 \%$ para CA $60-70$ respectivamente.

\subsection{Caracterización Dinámica}

En las Fig. 3 y 4 se presenta la evolución del módulo resiliente y la deformación permanente en la dirección vertical de las mezclas asfálticas modificadas con PVC. Se observa un incremento típico del módulo cuando se aumenta la frecuencia de carga y disminuye la temperatura del ensayo. La rigidez de las mezclas incrementa notablemente, y por lo tanto la resistencia a la deformación permanente, cuando se adiciona el PVC al CA. Para el caso de la temperatura de ensayo de $10^{\circ} \mathrm{C}$ el módulo de las mezclas modificadas incrementa aproximadamente entre un 58-113\% y 29 $69 \%$ con respecto a la mezcla convencional cuando se adiciona PVC entre $0,5-1,5 \%$ a los CA 80-100 y CA 60-70 respectivamente. Para 
temperaturas de $20^{\circ} \mathrm{C} \mathrm{y} 30^{\circ} \mathrm{C}$ estos incrementos son de $114-460 \%$ y $67-334 \%$ respectivamente.

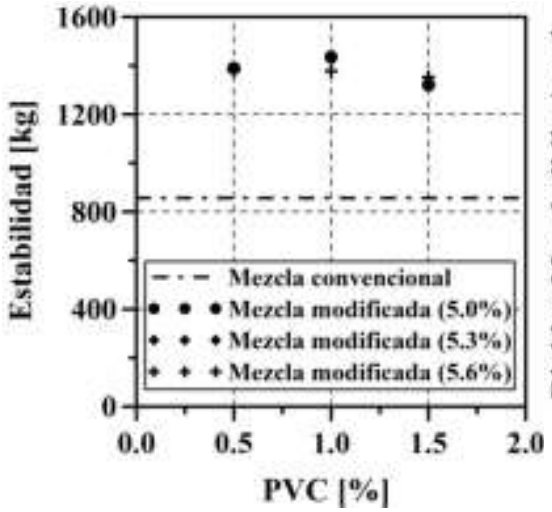

a)

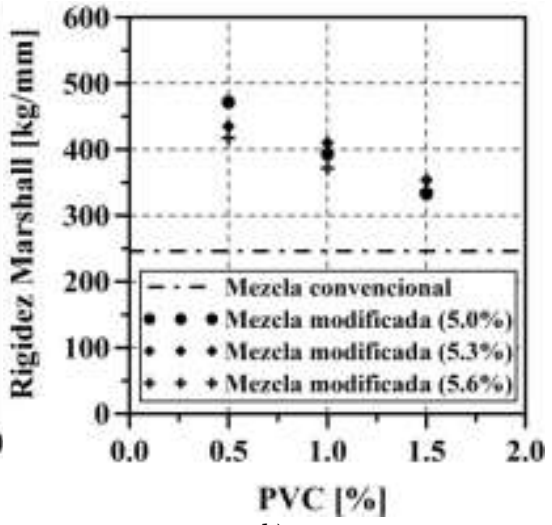

b)

Fig. 1. a) Estabilidad, b) rigidez Marshall vs. Porcentaje de desecho de PVC para mezclas modificadas MDC-2 empleando CA 80-100. Fuente: Autores

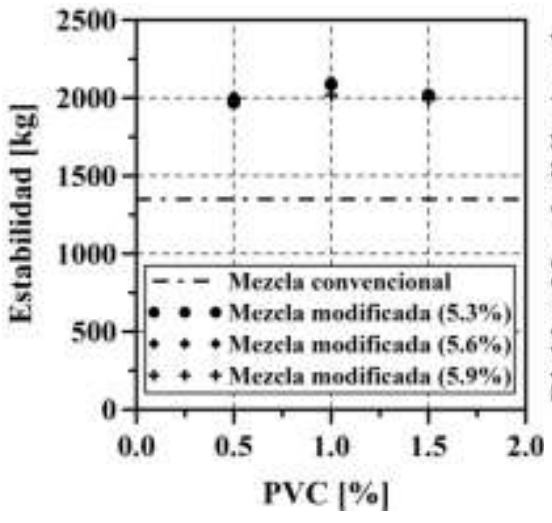

a)

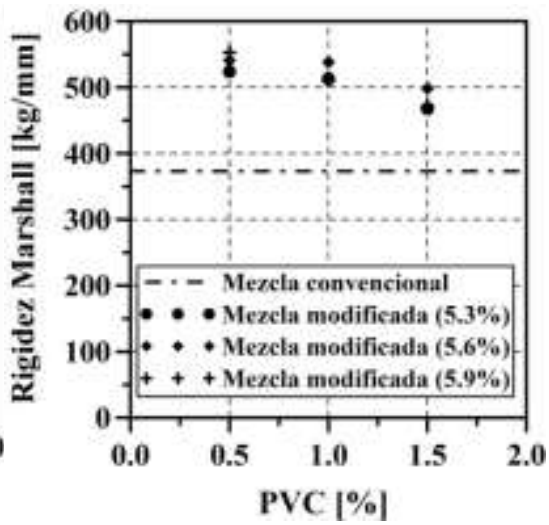

b)

Fig. 2. a) Estabilidad, b) rigidez Marshall vs. Porcentaje de desecho de PVC para mezclas modificadas MDC-2 empleando CA 60-70. Fuente: Autores

La deformación permanente disminuyó entre 25-41\% (CA 80100) y 11-27\% (CA 60-70) cuando se adicionó PVC entre 0,5-3,5\% respectivamente. Este aumento en rigidez y resistencia a la deformación permanente se debe a que con la incorporación del PVC 
[22] Evaluación de las Propiedlades Mecánicas de una Mezcla Densa en Caliente Modificada con un Desecho de PVC

al CA se obtiene un material más rígido y viscoso tal como se observa en las Fig. 5 y 6.

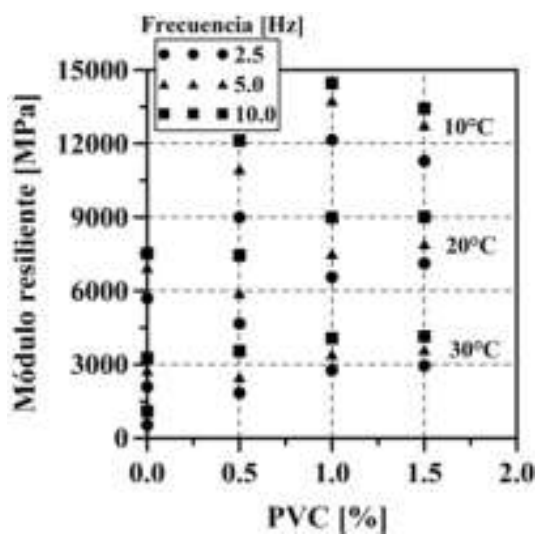

a)

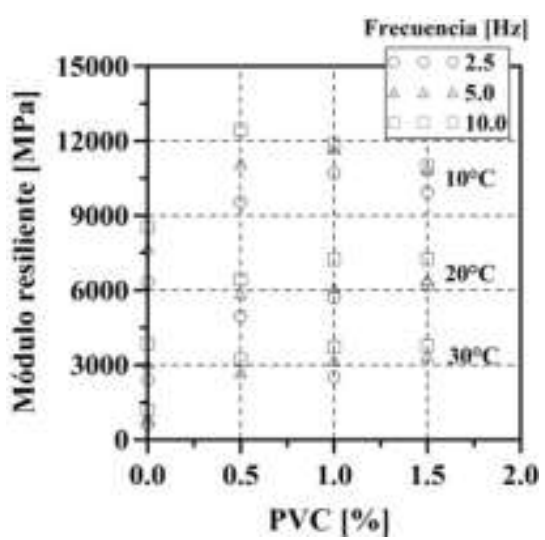

b)

Fig. 3. Evolución del módulo resiliente vs. Porcentaje de PVC para mezclas modificadas MDC-2 empleando a) CA 80-100 y b) CA 60-70. Fuente: Autores

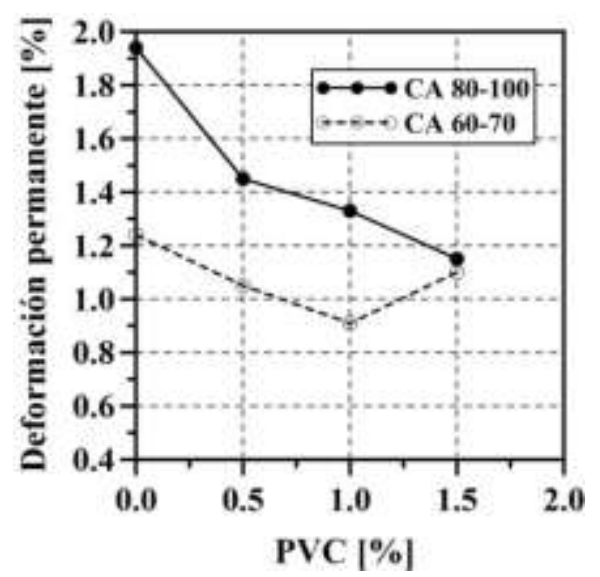

Fig. 4. Evolución de la deformación permanente vertical vs. Porcentaje de PVC para mezclas modificadas MDC-2 empleando CA 80-100 y CA 60-70.

Fuente: Autores 


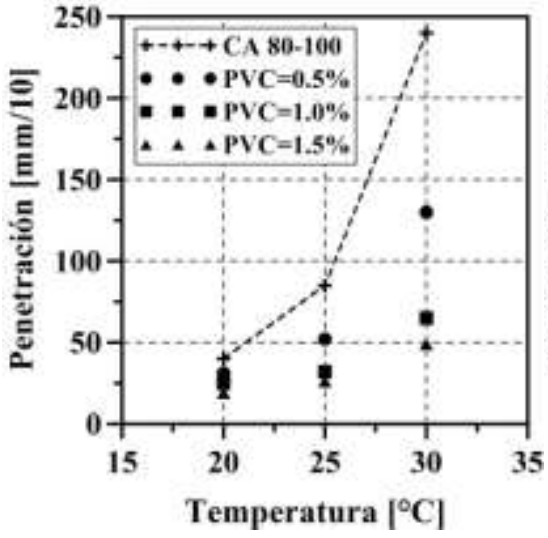

a)

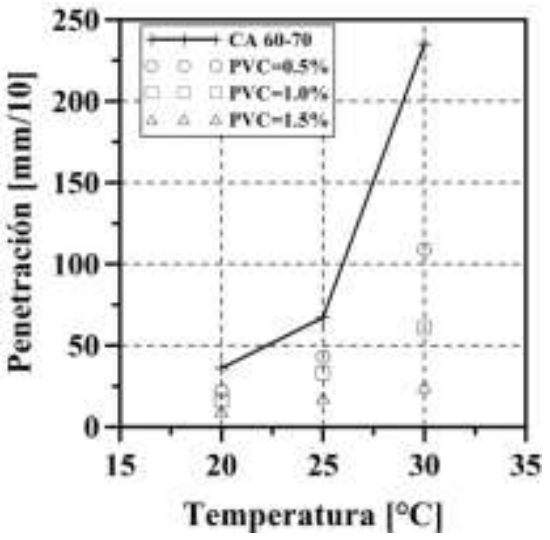

b)

Fig. 5. Penetración vs. temperatura del ensayo para a) CA 80-100 y b) CA 60-70. Fuente: Autores

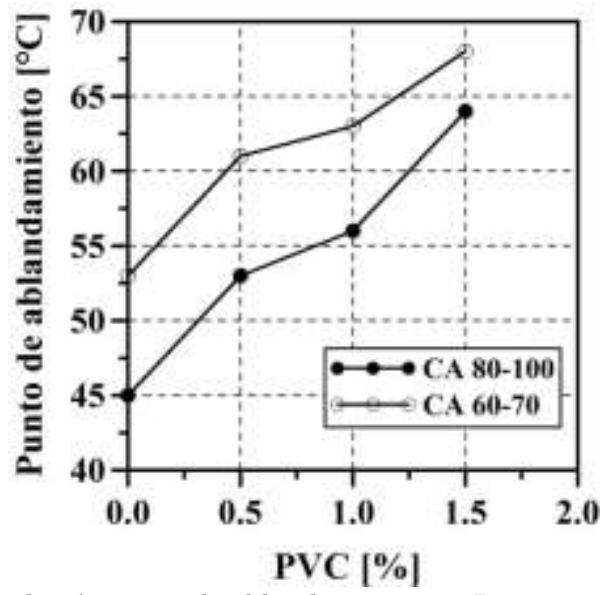

Fig. 6. Evolución punto de ablandamiento vs. Porcentaje de PVC.

Fuente: Autores

\subsection{Influencia del Medio Ambiente de la Ciudad de Bogotá D.C.}

Las mezclas modificadas sometidas al medio ambiente de la ciudad de Bogotá D.C., fueron fabricadas utilizando la siguiente composición: $5,3 \%$ de CA $80-100$ y $0,7 \%$ de PVC, y 5,6\% de CA 60 70 con $0,7 \%$ de PVC. En la Fig. 7 se presenta la evolución de los 
parámetros $E$ y $E / F$ de las mezclas asfálticas convencionales y modificadas con el tiempo ( $t$ en meses) de exposición al medio ambiente de la ciudad de Bogotá D.C.

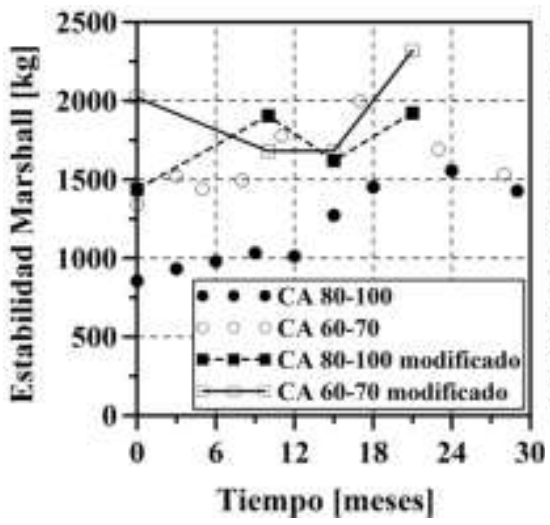

a)

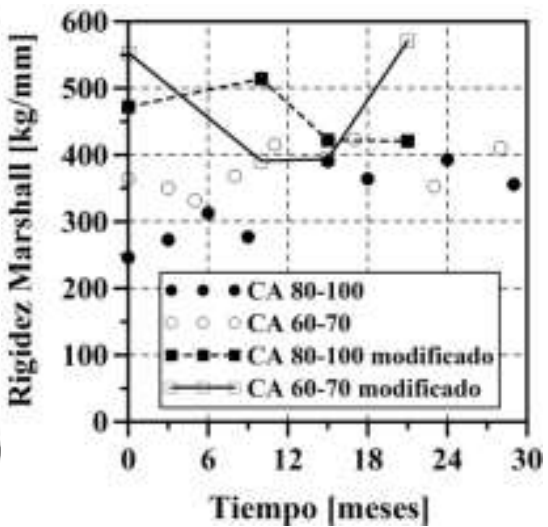

b)

Fig. 7. Evolución de a) estabilidad Marshall y b) E/F con el tiempo de envejecimiento, para mezclas convencionales y modificadas. Fuente: Autores

Se observa para las mezclas fabricadas con CA 80-100 y CA 6070 un incremento en la resistencia bajo carga monotónica con el tiempo de exposición. Para el caso de las mezclas fabricadas con CA 80-100 este incremento en $t=29$ meses es de $75 \%$ y $60 \%$ para $E$ y $E / F$ respectivamente. Para aquellas fabricadas con CA 60-70 en $t=17$ meses el incremento de $E$ es de $54 \%$ y luego disminuye en $t=28$ meses a $15 \%$. $E / F$ experimenta en $t=28$ meses un leve incremento de $7 \%$ y en algunos periodos de tiempo se observan intervalos de crecimiento y disminución de este parámetro mecánico. Lo anterior puede ser explicado por dos fenómenos que están ocurriendo en las mezclas simultáneamente: primero, el envejecimiento por oxidación que experimenta el cemento asfáltico, y por lo tanto las mezclas, cuando es solicitado a diferentes gradientes de temperatura y exposición a radiación ultra-violeta (UV) tal como ha sido ampliamente reportado por otros investigadores (Khalid, 2002; Airey, 2003; Said, 2005), y segundo, el agua en las mezclas produce pérdida de adherencia entre el agregado pétreo y el asfalto, lo cual genera un aumento del flujo y disminución de la relación $E / F$. 
Para el caso de las mezclas modificadas, el comportamiento que experimentan es totalmente contrario al de las convencionales. En los primeros 15 meses de exposición al medio ambiente, las mezclas modificadas con CA 60-70 experimentan una disminución (entre 17 a 29\%) en la resistencia bajo carga monotónica para luego incrementar hasta alcanzar valores similares e incluso superiores al de las mezclas sin envejecer. El decaimiento en la resistencia es debido a la generación de micro-fisuras que se producen a bajas temperaturas cuando las mezclas son rígidas (comportamiento frágil), luego estas micro-fisuras se estabilizan y el aumento en rigidez se debe a un fenómeno de envejecimiento y oxidación del asfalto. El fenómeno de micro-fisuración a bajas temperaturas ha sido ampliamente reportado por diversos investigadores (Kliewer et al., 1996; Sebaaly et al., 2002; Nesnas\&Nunn, 2006). Las mezclas modificadas con CA 80-100 experimentan en 21 meses, un aumento en $E$ de $34 \%$ y disminución en $E / F$ de $11 \%$.

En las Fig. 8 y 9 se presenta la relación $\left(E_{r} / E_{r o}\right)$ entre el módulo resiliente que se obtiene luego de someter las briquetas al ambiente durante distintos periodos de tiempo $\left(E_{r}\right)$ y el módulo resiliente inicial de las mezclas en un tiempo $t=0$ meses $\left(E_{r o}\right)$. Para el caso de las mezclas fabricadas con CA 80-100, el módulo alcanzado en $t=29$ meses aumenta entre un 64 y $185 \%$ (dependiendo de la temperatura y frecuencia del ensayo) con respecto al inicial, incrementando la resistencia a la deformación permanente (Fig. 8 y 10). Las mezclas con CA 60-70 experimentan una disminución máxima en su módulo de $35 \%$ en los primeros cinco meses de exposición, disminuyendo su resistencia a las deformaciones permanentes (ver Fig. 8 y 10). Luego el $E_{r}$ aumenta y experimenta en $t=28$ meses, valores que superan ligeramente el inicial, aumentando la resistencia a la deformación.

Estos resultados pueden ser expresados matemáticamente a través de (1) y (2) para las mezclas fabricadas con CA 80-100 y CA 60-70 respectivamente. Esta ecuación puede ser utilizada solamente para la mezcla analizada en el rango de tiempo evaluado (28-29 meses) ya que se debe prever un cambio del comportamiento de la misma a medida que aumente $t$. Las variables estado $k_{1}$ $k 3$ son obtenidas a través de regresión y no presentan un significado físico definido (ver Tabla 5). En la Fig. 8 se presenta el ajuste 
de los ensayos ejecutados empleando (1) y (2) y el coeficiente de correlación es $\mathrm{r}^{2}=0,79$.

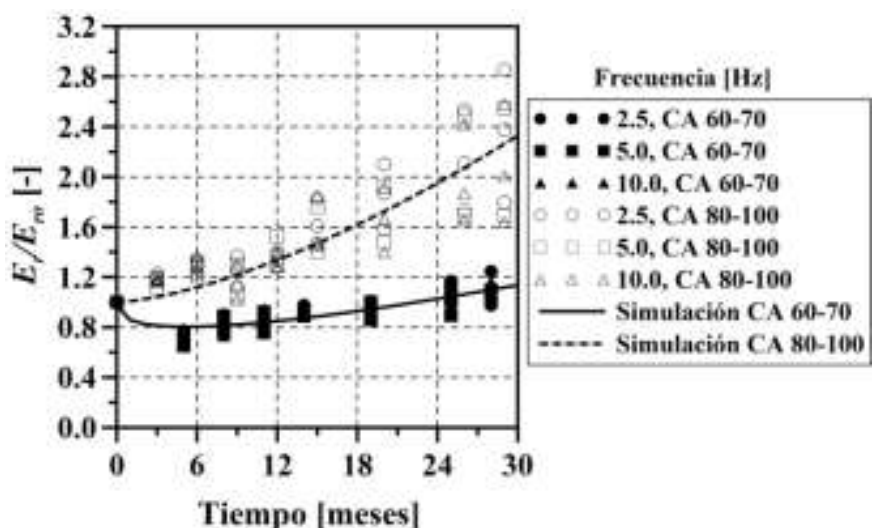

Fig. 8. Evolución de la relación entre el módulo resiliente $\left(E_{r}\right)$ y el módulo inicial $\left(E_{r o}\right)$ con el tiempo de envejecimiento $(t)$ para mezclas convencionales. Fuente: Autores

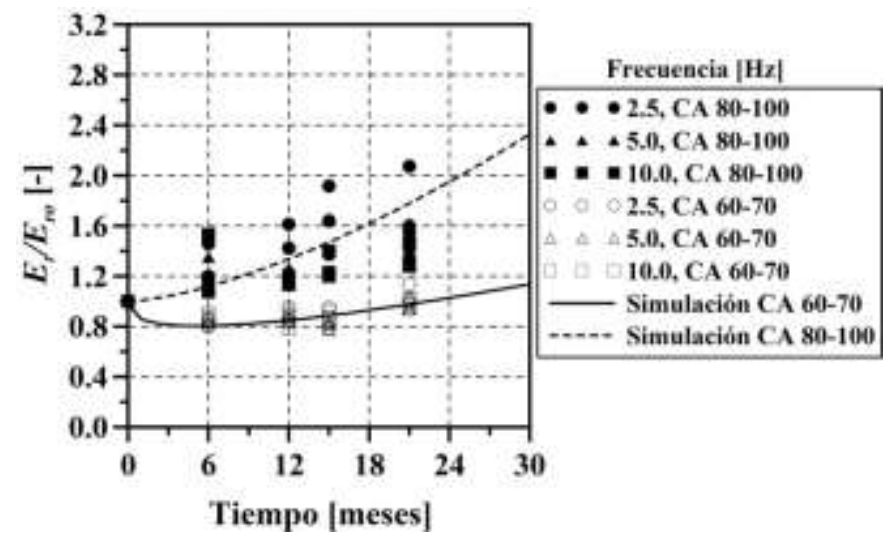

Fig. 9. Evolución de la relación entre el módulo resiliente $\left(E_{r}\right)$ y el módulo inicial $\left(E_{r o}\right)$ con el tiempo de envejecimiento $(t)$ para mezclas modificadas.

Fuente: Autores

Para el caso de las mezclas modificadas con PVC y fabricadas con CA 80-100 (ver Fig. 9), el módulo alcanzado en $t=21$ meses aumenta entre un 8 y 107\% (dependiendo de la temperatura y frecuencia del ensayo) con respecto al inicial y por lo tanto su 
resistencia a las deformaciones permanente incrementa (Fig. 10). Utilizando (1) se obtiene una correlación de $\mathrm{r}^{2}=0,67$. Las mezclas con CA 60-70 modificadas experimentan una disminución máxima en su módulo de $23 \%$ en los primeros 15 meses de exposición para luego aumentar y experimentar en $t=21$ meses, módulos que superan ligeramente el valor inicial, especialmente bajo temperatura de ensayo de $30^{\circ} \mathrm{C}$. Estos resultados de evolución del módulo son coherentes con los observados en la acumulación de las deformaciones permanentes (Fig. 10).

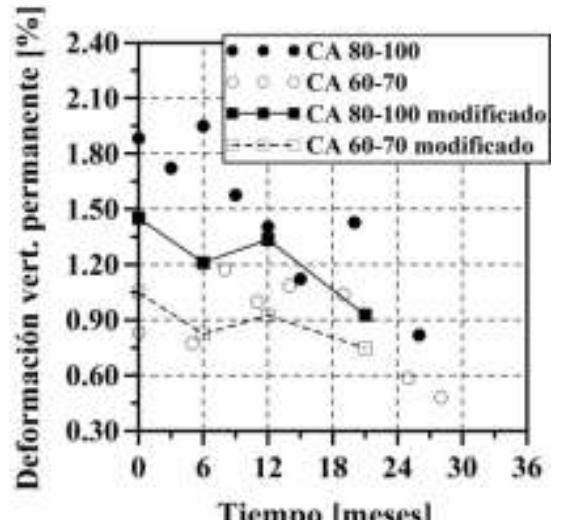

Fig. 10. Evolución de la deformación vertical permanente con el tiempo de envejecimiento $(t)$ para mezclas fabricadas con CA 80-100 y CA 60-70.

Fuente: Autores

Tabla 5. Variables para ajustar la rigidez de la mezcla MDC-2 en función del tiempo de exposición al medio ambiente. Fuente: Autores

\begin{tabular}{cccc}
\hline CA & $k_{1}$ & $k_{2}$ & $k_{3}$ \\
\hline $80-100$ & 0,0081 & - & - \\
$60-70$ & 0,028 & $-0,032$ & 0,15 \\
\hline
\end{tabular}

$\frac{E_{r}}{E_{r o}}=1+k_{1} t^{3 / 2}$, para CA $80-100$

$\frac{E_{r}}{E_{r o}}=1-\left(k_{1}+k_{2} t+k_{3} \sqrt{t}\right)$, para CA $60-70$ 


\section{CONCLUSIONES}

En general, las mezclas asfálticas modificadas con el desecho de PVC tienden a poseer un comportamiento rígido. A bajas temperaturas de servicio estas mezclas pueden tener un comportamiento frágil, llevando a pensar que tendrían un mejor desempeño en climas cálidos. Los valores de estabilidad y rigidez Marshall de las mezclas modificadas con CA 80-100 y CA 60-70 son mayores, para cualquier porcentaje de CA y PVC, en comparación con la mezcla convencional. El mejor comportamiento de las mezclas, con base en los ensayos de resistencia bajo carga monotónica, caracterización dinámica y viscosidad se presenta cuando se adiciona $0,7 \%$ de PVC (con respecto al peso total de la mezcla) al contenido óptimo de CA. Los módulos dinámicos de las mezclas modificadas con CA 80-100 son superiores a aquellos alcanzados por las convencionales, y los mayores incrementos se obtienen cuando la temperatura del ensayo aumenta. Lo anterior permite prever que el PVC como modificador de asfaltos puede ser un material que permita mejorar las características de rigidez y resistencia a las deformaciones permanentes de mezclas que sean utilizadas en climas cálidos. La resistencia que tienen los asfaltos modificados a fluir es mayor con respecto al convencional. Los valores de penetración del asfalto modificado permiten predecir menor ahuellamiento a altas temperaturas de servicio en comparación con los convencionales.

En este estudio se ejecutó adicionalmente una fase experimental destinada a medir la influencia de las condiciones climáticas de la ciudad de Bogotá D.C. sobre las propiedades mecánicas de una mezcla de concreto asfáltico tipo MDC-2 fabricada con CA 80-100 y CA 60-70, y modificadas con el desecho de PVC. Para el caso de las mezclas fabricadas con CA 80-100 y modificadas con PVC, se concluye que la rigidez bajo carga cíclica incrementa con la exposición al medio ambiente. Para el caso de las mezclas fabricadas con CA 60-70 y modificadas con PVC, en los primeros meses de exposición el módulo resiliente y la resistencia bajo carga monotónica disminuyen por fenómenos de micro-fisuración térmica y luego aumentan por efecto del envejecimiento del ligante asfáltico. 
Las fases futuras del proyecto deben medir propiedades como envejecimiento a corto y largo plazo de los asfaltos y resistencia a fatiga de las mezclas asfálticas. Adicionalmente para entender los cambios que ocurren en las propiedades físicas y mecánicas de las mezclas modificadas es necesario evaluar los cambios que experimentan las propiedades químicas de las mismas.

\section{REFERENCIAS}

Airey, G.D., (2003); State of the Art Report on Ageing Test Methods for Bituminous Pavement Materials, Int. Journal of Pavement Engineering, 4(3), 165-176.

CAG - Ciudadanía Ambiental Global, (2009); Basura al Aire Libre, http://www.cag.org.mx/16.html,Acceso 12 Octubre.

CEN - Committee European of Normalization, (2005); Bituminous Mixture Test Methods for Hot Mix Asphalts - Part25: Cyclic Compression Tests.

Chen, J-S., Liao, M-C., Shiah, M-S., (2002); Asphalt modified by StyreneButadiene-Styrene Triblock Copolymer: Morphology and Model, J. Material in Civil Eng.,14(3), 224-229.

Copeland, A.R., Youtcheff, J.S. Jr., Shenoy, A., (2007); Moisture Sensitivity of Modified Asphalt Binders: Factors Influencing Bond Strength, Transportation Research Record: Journal of the TRB, Issue number 1998, 18-28.

Fang, C., Li, T., Zhang, Z., Ping, D., (2008); Modification of Asphalt by Packaging Waste-Polyethylene, Polymer Composites, 29(5), 500-505.

IDU - Instituto de Desarrollo Urbano, (2009); Especificación Técnica para la Aplicación del Grano de Caucho Reciclado (Gcr) en Mezclas Asfálticas en Caliente (Vía Húmeda), Bogotá D.C., Colombia.

INVIAS - Instituto Nacional de Vías, (2007a); Especificaciones Generales de Construcción de Carreteras, Bogotá D.C (Colombia).

INVIAS - Instituto Nacional de Vías, (2007b); Normas de Ensayos de Materiales para Carreteras. V. I y II. Bogotá D.C (Colombia).

Jemison, H.B., Davison, R.R., Glover, C.J., Bullin, J.A., (1991); Evaluation of Standard Oven Tests for Hot-Mix Plant Aging, Transportation Research Record: Journal of TRB, Issue number 1323, 77-84. 
Kemp, G.R., Predoehl, N.H., (1981); A Comparison of Field and Laboratory Environments of Asphalt Durability, Proc. Assn. Asphalt Paving Technol., Vol. 50, 492-537.

Khalid, H.A., (2002); A New Approach for the Accelerated Ageing of Porous Asphalt Mixtures, Proc. of the Institution of Civil Engineers, 153(3), 171-181.

Kim, O.K., Bell, C.A., Wilson, J.E., Boyle, G., (1987); Development of Laboratory Oxidative Aging Procedures for Asphalt Cements and Asphalt Mixtures, Transportation Res. Record: Journal of the TRB, Issue number $1115,101-112$.

Kliewer, J.E., Zeng, H., Vinson, T.S., (1996); Aging and Low-Temperature Cracking of Asphalt Concrete Mixture, Journal of Cold Regions Engineering, 10(3), 134-148.

Lee, S-J., Amirkhanian, S-N., Shatanawi, K., Thodesen, C., (2008); Influence of Compaction Temperature on Rubberized Asphalt Mixes aqnd Binders, Canadian J. Civil Eng., 35(9), 908-917.

McQuillen, J.L., Takallou, H.B., Hicks, R.G., Esch, D., (1988); Economic Analysis of Rubber-Modified Asphalt Mixes, J. Transportation Engineering, 114(3), 259-277.

Migliori, F., Corté, J.F., (1998); Comparative Study of RTFOT and PAV Aging Simulation Laboratory Tests, Transp. Res. Record: J. of TRB, Issuenumber 1638, 56-63.

Ministerio de Transporte - MINTRANSPORTE - Oficina de Planeación, (2004); El Transporte en Cifras. Bogotá D.C., Colombia.

Ministerio de Transporte - MINTRANSPORTE - Dirección de Transporte y Tránsito, (2006); Parque Automotor de Transporte de Carga en Colombia. Bogotá D.C., Colombia.

Nesnas, K., Nunn, M.E., (2006); A Thermal Pavement Response Model for Top-Down Reflection Cracking in Composite Pavements, In: 85th Annual Meeting of the Transportation Research Board, paper No. 060127, Washington, D.C.

Olivares, F., Schultz, B., Fernández, M., Moro, B., (2009); RubberModified Hot-Mix Asphalt Pavement by Dry Process, Int. J. Pavement Engineering, 10(4), 277-288.

Papagiannakis, A.T., Lougheed, T.J., (1995); A Review of Crumb-Rubber Modified Asphalt Concrete Technology, Res. Report for Project T9902- 
09 "Rubber-Asphalt Study", Washington State Transp. Commission and U.S. Department of Transp.

Reyes, F. A., Figueroa, A. F., (2008); Uso de Desechos Plásticos en Mezclas Asfálticas - Síntesis de la Investigación Colombiana, Ed. Pontificia U. Javeriana, Bogotá D.C. (Colombia), 103 p.

Rondón, H.A., Reyes, F.A., Figueroa, A.S., Rodríguez, E., Real, C.M., Montealegre, T.A., (2008); Mezclas Asfálticas Modificadas en Colombia. Revista Infraestructura Vial, No. 19, 12-21.

Said, S.F., (2005); Aging Effect on Mechanical Characteristics of Bituminous Mixtures, Transportation Research Record: Journal of the TRB, Issue number 1901, 1-9.

Sebaaly, P.E., Lake, A., Epps, J., (2002); Evaluation of Low-Temperature Properties of HMA Mixtures, Journal of Transportation Engineering, 128(6), 578-586.

Shen, J., Amirkhanian, S., Tang, B., (2006); Influence of Accelerated Aging Test Temperature on The Properties of Binders, Int. J. of Pavement Eng., 7(3), 191-198.

Stastna, J., Zanzotto, L., Vacin, O., (2000); Damping of Shear Vibrations in Asphalt Modified with Styrene-Butadiens-Styrene Polymer, Transp. Res. Record, J. of TRB, Issue number 1728, 15-20.

Stastna, J., Zanzotto, L., Vacin, O. (2003); Viscosity Function in PolymerModified Asphalts, Journal of Colloid and Interface Science, 259(1), 200-207.

Verhasselt, A.F., (1997); Field Ageing of Bituminous Binders: Simulation and Kinetic Approach, In: Di Benedetto, H., Francken, L., Proc. Fifth International RILEM Symposium, Mechanical Tests for Bituminous Materials, 1(1), 121-128.

Welborn, J.Y., (1984); Physical Properties as Related to Asphalt Durability: State of the Art, Transportation Res. Record: Journal of theTRB, Issuenumber 999, 31-36. 
